\title{
Generalized Low-Density Parity-Check Codes for Optical Communication Systems
}

\author{
Ivan B. Djordjevic, Member, IEEE, Olgica Milenkovic, Member, IEEE, and Bane Vasic, Senior Member, IEEE
}

\begin{abstract}
This paper is concerned with investigating the performance of regular and irregular, randomlike and structured generalized low-density parity-check (GLDPC) codes for long-haul transmission. The proposed GLDPC codes outperform currently known turbo and low-density parity-check (LDPC) coding schemes with comparable parameters utilized in optical communication systems. For a GLDPC coding scheme with $23.6 \%$ redundancy, the largest so far reported coding gain of at least $11 \mathrm{~dB}$ (at $40 \mathrm{~Gb} / \mathrm{s})$ is demonstrated.
\end{abstract}

Index Terms-Forward-error correction (FEC), generalized low-density parity-check codes (GLDPCs), optical communications.

\section{INTRODUCTION}

$\mathbf{F}$ ORWARD-ERROR correction (FEC) [1]-[35] is becoming an increasingly important signal processing scheme for use in modern high-speed long-haul wavelength-division multiplexing (WDM) systems. This is due to the fact that FEC can provide an effective increase of the amplifier spacing, transmission distance, and the systems capacity. As an example, two FEC schemes are currently used in optical networking interface devices: one, consisting of parallel Reed-Solomon (RS) codecs, each operating at $2.5 \mathrm{~Gb} / \mathrm{s}$, and another, based on rate 1/2, block-length 1024 low-density parity-check (LDPC) codes [1], [3]. Practical multipurpose codecs employing proprietary multiedge-type LDPC code and block turbo code (BTC)-based FEC schemes were described for use in wireless and optical communication systems in [4] and [5]. The former scheme exhibits a gap of at most $1.5 \mathrm{~dB}$ to capacity, while the latter gives a coding gain of $10.1 \mathrm{~dB}$ with $24.6 \%$ overhead. The performance of turbo codes can be matched and improved by LDPC codes, which have additionally very small hardware complexity [6], [7]. However, LDPC decoding is based on a sub-optimal message-passing algorithm [8], and therefore one may expect to be able to further improve this coding scheme in terms of its distance to Shannon's limit. Generalized low-density parity-check (GLDPC) coding provides one possible way

Manuscript received May 27, 2004; revised January 19, 2005. This work is supported by the National Science Foundation (NSF) under Grant ITR 0325979 and Grant CCR 0208597.

I. B. Djordjevic is with the Department of Electrical and Computer Engineering, University of Arizona, Tucson, AZ 85721 USA, on leave from the Faculty of Computing, Engineering and Mathematical Sciences, University of the West of England, Bristol, U.K. (e-mail: ivan@ece.arizona.edu).

O. Milenkovic is with the Department of Electrical and Computer Engineering, University of Colorado, Boulder, CO 80303 USA (e-mail: milenkov@ colorado.edu).

B. Vasic is with the Department of Electrical and Computer Engineering, University of Arizona, Tucson, AZ 85718 USA (e-mail: vasic@ece.arizona.edu).

Digital Object Identifier 10.1109/JLT.2005.846892 to achieve this task and improve the overall characteristics of LDPC codes. These codes were first described by Tanner [9], and even though they seemed to offer both excellent minimum distance and low-complexity decoding, they remained unexplored until the recent work of Boutros et al. [10] and Lentmaier and Zigangirov [11].

When properly designed, GLDPC codes have very large minimum distance and exhibit performance approaching Shannon's limit. GLDPC decoding is based on a combination of simple and fast soft-input-soft-output (SISO) decoding of short, linear block codes, such as Hamming codes, $\mathrm{BCH}$ or Reed-Muller codes. To construct a GLDPC code, one has to replace each single parity-check equation of a global LDPC code by the parity-check matrix of a simple linear block code, known as the constituent or local code [10]-[16]. In the setting of Boutros et al. [10], [12], apart from the parity check matrix of the local code, several other code parameters have to be memorized, including the codeword length, the number of supercodes and a permutation matrix. For local codes of short length and high rate, trellis based maximum a posteriori probability (MAP) decoding, known as BCJR (Bahl-Cocke-Jelinek-Raviv) [17], or its low complexity version Max-Log-MAP [13], are used. Additionally, for Hamming and Reed-Muller codes one can utilize a simple MAP algorithm based on the modified Walsh-Hadamard transform proposed in [18], with complexity proportional to $N \log _{2} n$, where $n$ stands for the number of bits processed by constituent code and $N$ for the overall codeword length. Time efficient MAP decoding algorithms operating on local codes provide accurate estimates for the variable nodes in the global LDPC graph after a very small number of iterations (usually less than ten). The low complexity of encoding and especially decoding makes FEC schemes based on GLDPC codes an attractive option for high-speed optical transmission.

Two classes of generalized LDPC codes are considered in this paper: randomlike, as well as a novel class of structured GLDPC codes. Here, the properties refer to the choice of the parity-check matrix of the global LDPC code. In both cases, we will investigate GLDPC codes for which the local codes are Hamming codes or structured, short length LDPC codes themselves. For the first class of codes, decoding on the local level is accomplished through the use of an appropriate number of BCJR decoders operating in parallel; for the second class of codes, low-complexity decoding based on suboptimal message-passing algorithm (MPA) is used instead.

The performance of the proposed codes is assessed in a realistic simulation environment [6], [19], that includes impairments originating from amplified spontaneous emission (ASE) noise, pulse distortions due to Kerr nonlinearities 
(self-phase modulation, cross-phase modulation, four-wave mixing) and stimulated-Raman scattering (SRS), chromatic dispersion (group-velocity dispersion (GVD), second-order GVD), crosstalk effects, and intersymbol interference (ISI). For a given code rate of 0.809 (23.6\% redundancy) the largest so far reported coding gain for an FEC scheme of the order of at least $11 \mathrm{~dB}$ is found, therefore outperforming the turbo coding scheme of larger redundancy $(24.6 \%)$ described in [5] by more than $1 \mathrm{~dB}$.

\section{CODE DESCRIPTION}

In this section, we will use the most basic notions regarding LDPC coding and decoding without detailed explanation. For a thorough exposition of this coding technique, the interested reader is referred to [28].

Depending on the structure of the local components, GLDPC codes may be classified as follows:

1) GLDPC codes with algebraic local codes of short length, such as Hamming codes, BCH codes, RS codes or Reed-Muller codes [20];

2) GLDPC codes for which the local codes are high-rate regular or irregular LDPC codes with large minimum distance;

3) Fractal GLDPC codes in which the local code is in fact another GLDPC code.

Each of these three constructions offers specific advantages. For example, in the first scenario, the MAP decoders of the local codes allow for fast propagation of accurate estimates for the LDPC variables. Consequently, the number of global iterations needed to achieve very low BERs over the LDPC graph is small, usually below ten. The second construction is very well suited for longer length codes. Since the local components in this case can have large length and therefore large minimum distance as well, the minimum distance of the resulting GLDPC codes is very high. The third approach is an attractive idea in terms of possible practical partly-parallel very large scale integration (VLSI) implementations [21], since the same code structure can be reused on different levels.

In general, one seeks GLDPC codes for which the local codes have large minimum distance and a rate as high as possible, and for which the global code has very large girth. This is a consequence of the following lower bound on the minimum distance $D$ of a GLDPC code [9], found in (1) at the bottom of the page, where $g$ and $J$ denote the girth-i.e., length of the shortest cycle of the global code graph - and the column weight of the global code, respectively; in addition, $d$ is used to denote the minimum distance of the local code. Obviously, large girth leads to an exponential increase in the minimum distance, while large values of $d$ lead to an increase of the basis of this exponential function. GLDPC codes can be designed in such a way (see the references in Sections II-B and II-C) that their minimum distance $D$ grows linearly with the code length.

The codes proposed by Boutros [10] and Lentmaier and Zigangirov [11], described next, are based on randomlike global LDPC code in which the simple check nodes of the corresponding bipartite graph are replaced by local Hamming codes. We will refer to these codes as the $\boldsymbol{B}$ and $\boldsymbol{L} \boldsymbol{Z}$ GLDPC codes, respectively. Lower bound on minimum distance of such GDLPC codes were described in [11].

\section{A. The Construction of $\boldsymbol{B}-G L D P C$ Codes}

The parity-check matrix of a GLDPC code proposed by Boutros et al. [10], [12], is a sparse matrix $H$ constructed in the following manner. The matrix $H$ is partitioned into $W$ submatrices $H_{1}, H_{2}, \ldots, H_{W} \cdot H_{1}$ is a block-diagonal matrix obtained from an identity matrix, with ones on the main diagonal replaced by the parity-check matrix $H_{0}$ of a local code $C_{0}(n, k)$. Each of the $H_{j}$ submatrices is derived from $H_{1}$ through a random column permutation $\pi_{j-1}$, as shown hereafter:

$$
\begin{aligned}
H & =\left[\begin{array}{llll}
H_{1}^{T} & H_{2}^{T} & \ldots & H_{W}^{T}
\end{array}\right]^{T} H_{1}=\left[\begin{array}{llll}
H_{0} & & & 0 \\
& H_{0} & & \\
& & \ldots & \\
0 & & & H_{0}
\end{array}\right] \\
H_{j} & =\pi_{j-1}\left(H_{1}\right), j=2, \ldots, W .
\end{aligned}
$$

If the rate of the constituent code $C_{0}(n, k)$ is $r=k / n$, the designed rate of the GLDPC code is

$$
R=\frac{K}{N} \geq 1-W\left(1-\frac{k}{n}\right)
$$

with $K$ and $N$ denoting the dimension and code-length of the resulting GLDPC code, respectively. Therefore, this type of GLPDC code, denoted as $\operatorname{GLDPC}(N, W, n)$, is the intersection of $W$ super-codes $C_{1}, C_{2}, \ldots, C_{W}$ whose parity-check matrices are $H_{1}, H_{2, \ldots}, H_{W}$, respectively. It has been shown [10], [12]-[15] that $W=2$ leads to excellent BER performance. In this paper, we will also consider codes with $W \geq 3$ and irregular codes for which the average value of $W$ is bounded from above by two.

Zhang and Parhi proposed both an efficient encoding algorithm [14] and low-complexity decoding [13] based on MaxLog-MAP version of BCJR for this type of GLDPC codes. Lowcomplexity decoding of GLDPC codes is an active research topic and several improved performance and low-complexity decoding algorithms were recently proposed in [15], [16].

$\boldsymbol{B}$-GLDPC codes can effectively be decoded using the following iterative decoding scheme [10], [12], [13]: For each bit we calculate its reliability from a given sample assuming that it belongs to the super-code $C_{1}$ with parity-check matrix $H_{1} . N / n$ SISO decoders work in parallel on independent $N / n$ constituent

$$
D \geq\left\{\begin{array}{cl}
d \frac{[(d-1)(J-1)]^{(g-2) / 4}-1}{(d-1)(J-1)-1}+\frac{d}{J}[(d-1)(J-1)]^{(g-2) / 4} & , \text { for } \frac{g}{2} \text { odd } \\
d \frac{[(d-1)(J-1)]^{g / 4}-1}{(d-1)(J-1)-1}, & \text { for } \frac{g}{2} \text { even }
\end{array}\right.
$$


codes $C_{0}$ of super-code $C_{1}$. For every coded bit an $a$ posteriori probability and an extrinsic probability are computed. The extrinsic probabilities from super-code $C_{1}$ are fed to $N / n$ constituent codes $C_{0}$ of super-code $C_{2}$. The procedure is repeated for every super code: extrinsic probabilities of super-code $C_{2}$ are fed to $N / n$ constituent codes $C_{0}$ of super-code $C_{3}, \ldots$, while the extrinsic probabilities of super-code $C_{W-1}$ are fed to $N / n$ constituent codes $C_{0}$ of super-code $C_{W}$ (these steps define one iteration). The procedure is terminated either when a pre-determined number of iterations is reached or when a valid codeword is generated. Notice that for $W=2$ the GLDPC code may be considered as the generalization of a turbo decoder in which two BCJR algorithms are employed for each super-code [13]. In the case of GLDPC codes, the SISO decoding of upper or lower super-code is performed by employing $N / n$ identical low-complexity BCJR decoders (each operating on $H_{0}$ and different part of a codeword in parallel). The complexity can be further decreased by employing simple MAP decoding algorithm proposed in [18] instead of the original BCJR algorithm [17]. By employing the algorithm for efficient removal of cycles of length four proposed by the authors in [24] for algebraic codes with more complicated structure, binary $\mathrm{BCH}$ can also be efficiently decoded using a message-passing algorithm instead of a more complex BCJR algorithm.

Unfortunately, the performance of the described coding scheme strongly depends on the choice of the permutations $\pi_{i}$, as illustrated in Figs. 2 and 3; one may have to use results from the theory of $S$-random interleaving in order to fully analyze the bit-error rate (BER) characteristic of the $B$-GLDPC scheme [25], [26].

\section{B. The LZ-Construction of GLDPC Codes}

Another approach for designing of GLDPC codes is to replace ones within every row of the parity-check matrix of a global LDPC code by different columns from a local Hamming or some other algebraic code [11]. Since decoding is based on the BCJR algorithm applied to the local codes, this construction method is limited to the use of Hamming and possibly Reed-Muller local codes, whose minimum distance or rate is rather small.

\section{Structured Regular and Irregular GLDPC Codes}

The $\boldsymbol{L Z}$ GLDPC scheme can also be used in conjunction with a structured global code. It is also straightforward to make the global code irregular by erasing a number of predefined nonzero entries in the parity-check matrix and therefore increasing the rate of the code. We propose two possible new code design approaches below. The first idea is to either retain two block-rows of permutation matrix based codes, and then selectively erase ones from the blocks to achieve a given variable degree distribution. The second one is to use a modification of Latin squarebased structured LDPC codes, first described in a slightly different form by the authors in [28].

With this goal in mind, let $\omega$ be a primitive element of a finite field $G F\left(q^{m}\right), m \geq 2$ [29]. Define a set $S$ of integers by

$$
S=\left\{a: 0 \leq a<q^{m}-1, \omega^{a}+\omega \in G F(q)\right\}
$$

Let

$$
\begin{aligned}
H_{G} & =\left[\begin{array}{ccccc}
P^{i_{1}} & P^{i_{2}} & \ldots & P^{i_{q-1}} & P^{i_{q}} \\
P^{i_{q}} & P^{i_{1}} & \ldots & P^{i_{q-2}} & P^{i_{q-1}}
\end{array}\right], \\
P & =\left[\begin{array}{ccccc}
0 & 1 & 0 & \ldots & 0 \\
0 & 0 & 1 & \ldots & 0 \\
\ldots & & & & \ldots \\
1 & 0 & 0 & \ldots & 0
\end{array}\right], \quad I=P^{0}
\end{aligned}
$$

be the parity-check matrix of the global code, where $S=$ $\left\{i_{1}, \ldots, i_{s}\right\}$. For such a parity-check matrix structure a result by Fan [30] can be used to determine possible cycle formations in the code graph. The results in [30] show that a cycle of length $2 l$ exists in a code graph with a parity-check matrix $H$ composed of circulant permutation matrices $P^{i_{j}}$ if and only if one can find a closed path through the matrix $H$ for which the exponent of the matrices $\mathrm{P},\left\{i_{1}, i_{2}, \ldots, i_{2 l}\right\}$, satisfy $i_{1}+\cdots+i_{l}=i_{l+1}+\cdots+i_{2 l} \bmod N$, where $N$ denotes the dimension of the permutation matrices.

Example 1: Consider the matrix $H$ shown below, with $N=3$.

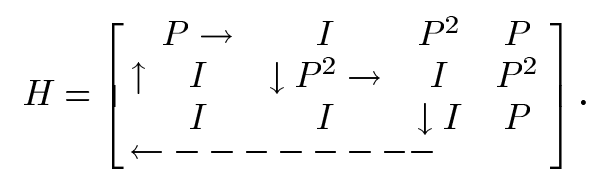

The arrows in $H$ indicate a closed path for which the exponents satisfy $1-0+2-0+0-0 \bmod 3=3 \bmod N=0$. Hence the path itself corresponds to three different cycles of length six in the code graph.

Theorem 1: Let $i_{l}, 1 \leq l \leq q, q \geq 5$ be elements of the set $S$ constructed according to (4), and with a given parameter $m \leq 5$.Then the LDPC code specified by $H_{G}$ has girth at least $2 m+2$.

Proof: It is straightforward to see that there cannot be any cycles of length four in any code constructed using the set defined by (4). If $m>2$, in order to have a cycle of length six in the code, there must exist six elements of the set $S$, say $\left\{i_{1}, i_{2}, \ldots, i_{6}\right\}$ such that without loss of generality

$$
i_{1}-i_{2}=\left(i_{3}-i_{4}\right)+\left(i_{5}-i_{6}\right) \bmod q^{4}-1 .
$$

This is equivalent to the requirement that

$$
\omega^{i_{1}-i_{2}}=\omega^{i_{3}-i_{4}} \cdot \omega^{i_{5}-i_{6}},
$$

or

$$
\omega^{i_{1}+i_{4}+i_{6}}=\omega^{i_{2}+i_{3}+i_{5}}
$$

The last equation implies the existence of six different integers $b_{1}, b_{2}, \ldots, b_{6} \in G F(q)$, for which it holds that

$\left(b_{1}-\omega\right) \cdot\left(b_{4}-\omega\right) \cdot\left(b_{6}-\omega\right)=\left(b_{2}-\omega\right) \cdot\left(b_{3}-\omega\right) \cdot\left(b_{5}-\omega\right)$.

This leads to the conclusion that $\omega$ is a root of an equation of degree at most two with coefficients over $G F(q)$, contradicting the starting assumption that $\omega$ is a primitive element of the field $G F\left(q^{m}\right)$ for $m>2$. All the other cases (up to the girth value 12) can be ruled out in a similar manner.

$Q E D$ 
The construction described above allows for designing global codes with a very simple structure and large values of girth. But, as will be demonstrated in the example below, if one desires to achieve a high code rate with such a construction, the code length has to be very large as well.

Example 2: If $q=7, m=4$, and the local code is chosen to be a Hamming [7], [4], [3] code, the underlying GLDPC code has minimum distance 33 , according to (1), with $J=2$. The length of the GLPC code is 16800 , while its rate is only $1 / 7$. On the other hand, if $q=17, m=2$ and only fifteen entries of $S$ are retained, the code length is 4320 , the code rate is $7 / 15$, while the minimum distance is at least 15 .

Another construction that results in global codes with relatively short length and girth at least eight is described below.

Theorem 2: Let $t$ be a fixed positive integer, and let $H$ be a parity check matrix with $t+1$ block-rows and $t(t+1) / 2$ block-columns, where each block is of dimension three. If the blocks in $H$ are specified according to the following two rules:

1) The first $t$ blocks in the first block-row are identity matrices, and all except the blocks at the main diagonal and beneath the first block-row are zeros; the entries at the diagonal itself are basic circulant permutation matrices $P$;

2) All the remaining entries in the matrix are obtained by consecutively deleting the last block-column of the submatrix described in the previous step and shifting the rows down in a circular fashion,

then the global code defined by $H$ has girth at least eight.

Proof: In order to clarify the method for constructing the parity-check matrix, we start with an example for $t=4$ as follows:

$$
\begin{aligned}
H & =\left[\begin{array}{llllllllll}
I & I & I & I & 0 & 0 & 0 & 0 & 0 & 0 \\
P & 0 & 0 & 0 & I & I & I & 0 & 0 & 0 \\
0 & P & 0 & 0 & P & 0 & 0 & I & I & 0 \\
0 & 0 & P & 0 & 0 & P & 0 & P & 0 & I \\
0 & 0 & 0 & P & 0 & 0 & P & 0 & P & P
\end{array}\right] \\
I & =P^{0}, \operatorname{dim}(P)=3 .
\end{aligned}
$$

The only way to form a six-cycle in a matrix described by $H$ is to trace through six blocks as follows:

$$
\left[\begin{array}{ccccc}
I & \ldots & I & \ldots & \ldots \\
\ldots & \ldots & \ldots & \ldots & \ldots \\
P & \ldots & \ldots & \ldots & I \\
\ldots & \ldots & \ldots & \ldots & \ldots \\
\ldots & \ldots & P & \ldots & P
\end{array}\right] .
$$

The sum of the exponents (with alternating sign changes) on this path is -1 , and therefore in violation of Fan's rule. $\quad \boldsymbol{Q E D}$

The codes described in Theorem 2 have length $3 t(t+1) / 2$, row weight $t$, and column weight 2 . For $\mathrm{t}=63$, the code length is 6048 , and the GLDPC code with a local Hamming code has rate 0.81 .

Regular GLDPC obtained from all the constructions described above usually have the drawback of resulting in a small or only moderate overall code rate. In order to increase the code rate, one can use irregular global codes, and in this case $J$ in (1) is to be replaced by the average variable node degree in the global graph. The regular codes described above can easily be made irregular by deleting certain permutation blocks. The two examples described below illustrate this idea.

Example 3: Consider the code described in Theorem 1 for the case that $q=27, m=2$, and that the first twelve blocks in the first block-row and the last twelve blocks in the second block row are set to zero. Then the average column weight of the global code is $J=1.11$, leading to a GLDPC code rate of $1-1.11(1-11 / 15)=0.7$. The length of this code is 19 656. Despite this seemingly large code length, the complexity of storing the description of the code and to perform decoding is extremely small. This is due to the fact that the local code is a [15], [11], [3] Hamming code and that effectively only 81 variables of these codes are exchanging information between each other.

Example 4: Consider the code described in Theorem 2 for the case that $t=70$; the code length in this case is 7455. One can delete the last seven submatrices of $H_{G}$ and set to zero seven $I$ blocks in each of the first 63 rows. This gives rise to a global code of length 7371 , and average variable degree 1.82 . The rate of the resulting GLDPC in this case is 0.83 .

Of course, one could use other forms for $H_{G}$ in order to achieve a trade-off between performance, rate and decoding complexity. For example, the codes in [28] with girth 20 and column weight two are a good candidate for this type of construction. Good irregular distributions for GLDPC codes can be found by using EXIT chart analysis, as described in [31].

\section{Fractal GLDPC Codes}

In fractal GLDPC codes, the local codes form several nested layers. More specifically, a fractal GLDPC code is defined as a GLDPC code for which the local code is itself a GLDPC, and where this construction can be extended throughout several levels.

Since the implementation of several nested layers may result in code rates unacceptably low for high speed optical transmission, we restrict our attention only to the class of GLDPC codes in which both local and global codes are LDPC codes, as illustrated in Fig. 1. As evident from (1), the global code should have the girth as large as possible, while the local code should have a large minimum distance. Since the low-complexity message passing algorithm is implemented for both global and local codes, the usage of local LDPC codes of minimum distance of at least 10 and global codes of high girth results in excellent BER performance, as will be shown in Section IV.

For the sake of completeness, we will next outline the employed decoding algorithm for fractal GLDPC codes.

Let $x=\left(x_{v}\right)_{1 \leq v \leq n}$ be a codeword, and let $H_{G}=\left(h_{s, v}\right)_{b x n}$ be the parity-check matrix of a global code (with $b$ and $n$ being the dimensions of $H_{G}$ ). Each row $s$ of this matrix represents a subcode node and every column $v$ a variable (bit) node in corresponding bipartite graph. An edge connecting vertices $s$ and $v$ exists if $h_{s, v}=1$, i.e., if variable $v$ participates in subcode $s$.

A priori information of the bit at position $v, \mu_{v}^{(0)}$, is determined as

$$
\mu_{v}^{(0)}=\log \left[\frac{P_{r}\left(x_{v}=0 \mid \mathbf{y}\right)}{P_{r}\left(x_{v}=1 \mid \mathbf{y}\right)}\right]
$$

with $\boldsymbol{y}$ denoting the received codeword. 


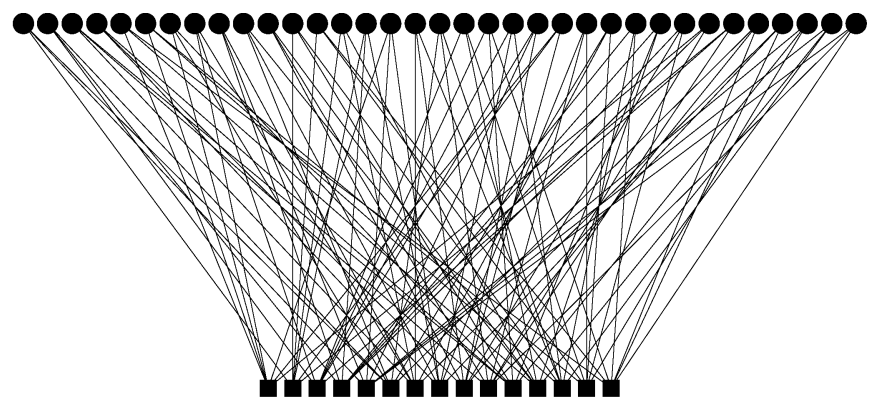

(a)

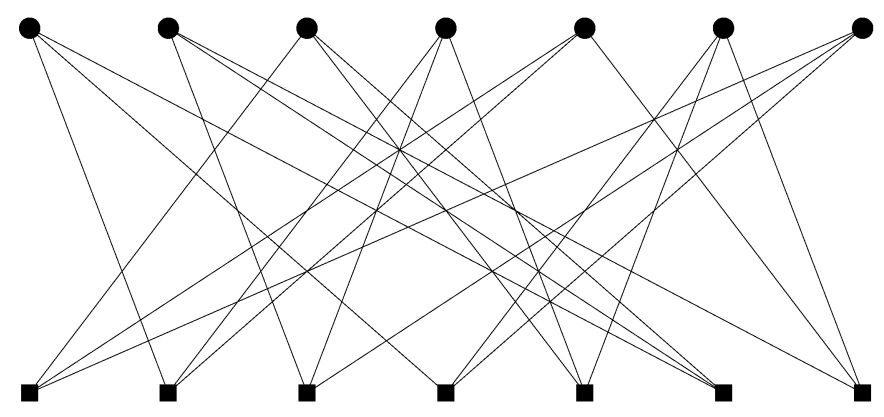

(b)

Fig. 1. Graph structure of GLDPC codes: (a) global code and (b) local code. Subcode node degree (7) in global code is identical with number of variable nodes in local code. Both global and local code were designed using our construction [33].

The messages passed from variable node $v$ to subcode node $s$ in the bipartite graph, $\lambda_{v, s}^{(0)}$, are initialized to $\mu_{v}^{(0)}$. In the $j$-th iteration, the messages to be passed from subcode node $s$ to bit node $v, \Lambda_{s, v}^{(j)}$, are obtained as the outputs of corresponding specialized low-complexity MAP or BCJR decoder, operating on the local code described by the parity-check matrix $H_{L}$. Obviously, the subcode node $s$ degree must be identical to the number of columns in $H_{L}$. The positions of ones in the $s$-the row of $H_{G}$ determine the variables passed to the local MPA or BCJR decoder. Messages to be passed from bit node $v$ to subcode node $s, \lambda_{v, s}^{(j)}$, are updated according to

$$
\lambda_{v, s}^{(j)}=\mu_{v}^{(0)}+\sum_{s^{\prime} \neq s} \Lambda_{s^{\prime}, v}^{(j)} .
$$

The last step in iteration $j$ is to compute updated log-likelihood ratios $\mu_{v}^{(j)}$ according to

$$
\mu_{v}^{(j)}=\mu_{v}^{(0)}+\sum_{s} \Lambda_{s, v}^{(j)} .
$$

For each bit $x_{v}$ the estimation is made according to

$$
\hat{x}_{v}=\left\{\begin{array}{ll}
1, & \text { if } \mu_{v}^{(j)}<0 \\
0, & \text { otherwise }
\end{array} .\right.
$$

Decoding halts when a valid codeword $\left(\sum_{v} h_{s, v} \hat{x}_{v}=0\right)$ or a predetermined maximum number of iteration has been reached.

\section{SySTEM MODEL DESCRIPTION}

The system of interest in this paper is a wavelength division multiplexing (WDM) system. The continuous-wave laser signals at different wavelengths are modulated using independently encoded electrical streams and a Mach-Zehnder (MZ) modulator, WDM multiplexed and transmitted over the same fiber. The carrier-suppressed RZ (CSRZ) modulator employed is composed of a laser diode, two MZ intensity modulators (the first serving as modulator, the second as a NRZ to RZ converter), a PRBS generator and an encoder. The optical signal at the receiver side is split into separate channels by using an optical demultiplexer. Erbium-doped fiber amplifiers (EDFA) and dispersion compensating fibers (DCF) are deployed periodically to compensate the loss and accumulated dispersion of the standard single mode fiber (SMF). The direct detection receiver observed is composed of a WDM demultiplexer, a p-i-n photodiode, an electrical filter, and a sampler followed by a decoder. An EDFA is used as a preamplifier.

The propagation of a signal through the transmission media is modeled by a nonlinear Schrödinger equation [6], solved using the split-step Fourier method.

For more details on transmission system model implemented reader is referred to our previous papers [6], [19].

In optical communication systems, it is common practice to use the $Q$ factor as a figure of merit, rather than the signal-tonoise ratio, and in this paper we will follow this convention.

\section{PERformance AnAlysis}

A WDM system with $40 \mathrm{~Gb} / \mathrm{s}$ bit-rate per channel and a channel spacing of $100 \mathrm{GHz}$ is considered. It is assumed that the observed channel is located at $1552.524 \mathrm{~nm}(193.1$ $\mathrm{THz}$ ) and that there exists a nonnegligible interaction with six neighboring channels. The dispersion map is composed of 25 spans of length $L=48 \mathrm{~km}$, consisting of $2 L / 3 \mathrm{~km}$ of $D+$ fiber followed by $L / 3 \mathrm{~km}$ of $D$ - fiber, with precompensation of $-320 \mathrm{ps} / \mathrm{nm}$ and corresponding postcompensation. The $Q$ factor is additionally decreased by noise loading. The $\mathrm{D}+$ fiber parameters are as follows; dispersion of $20 \mathrm{ps} /(\mathrm{nm} \cdot \mathrm{km})$, dispersion slope of $0.06 \mathrm{ps} /\left(\mathrm{nm}^{2} \cdot \mathrm{km}\right)$, effective cross-sectional area equal to $110 \mu \mathrm{m}^{2}$ and loss equal to $0.19 \mathrm{~dB} / \mathrm{km}$. The corresponding $D$ - fiber parameters are $-40 \mathrm{ps} /(\mathrm{nm} \cdot \mathrm{km})$, $-0.12 \mathrm{ps} /\left(\mathrm{nm}^{2} \cdot \mathrm{km}\right), 50 \mu \mathrm{m}^{2}$ and $0.25 \mathrm{~dB} / \mathrm{km}$, respectively. The nonlinear Kerr coefficient is set to $2.6 \times 10^{-20} \mathrm{~m}^{2} / \mathrm{W}$. The average power per channel is set to be $0 \mathrm{dBm}$, and a carrier-suppressed RZ signal format is assumed. The influence of optical and electrical filters is taken into account as well. The channel characteristics modeled according to our previous papers [6], [19] are used to create samples fed to iterative decoder. Using the expression

$$
\text { Uncoded_BER }=\left(\frac{1}{2}\right) \quad \operatorname{erfc}\left(\frac{Q}{\sqrt{2}}\right)
$$

the BER of uncoded signal is converted to $Q$ factor to make the results comparable with previously reported ones.

Results of simulations for $\boldsymbol{B}$-GLDPC codes are given in Figs. 2 and 3, for two different constituent codes. The GLDPC (3969,3213,0.809) code from Fig. 3 ("triangle curve" in Fig. 4) although of slightly smaller redundancy $(23.6 \%)$ compared to the best turbo code result proposed so far for optical transmission systems [5], demonstrates a coding gain of about $11.5 \mathrm{~dB}$ (at BERs below $10^{-12}$ ), the best results reported so far. Notice that the results reported in [5] are obtained experimentally, and 


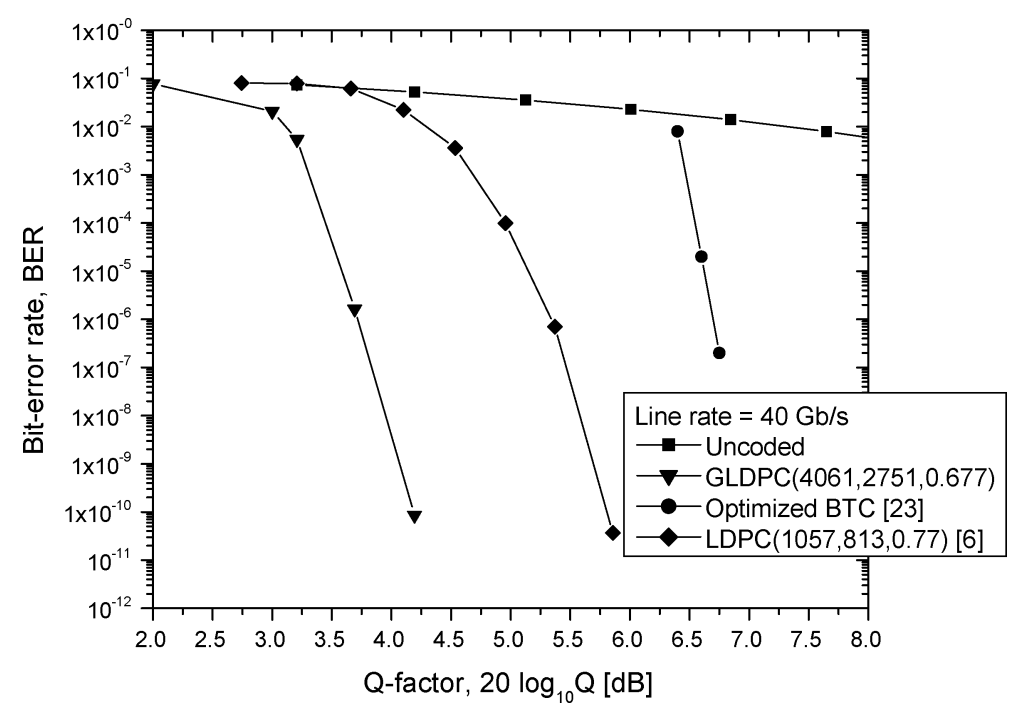

Fig. 2. BER performance of GLDPC codes with Hamming $(31,26)$ constituent code at $40 \mathrm{~Gb} / \mathrm{s}$ (line rate) (ten iterations).

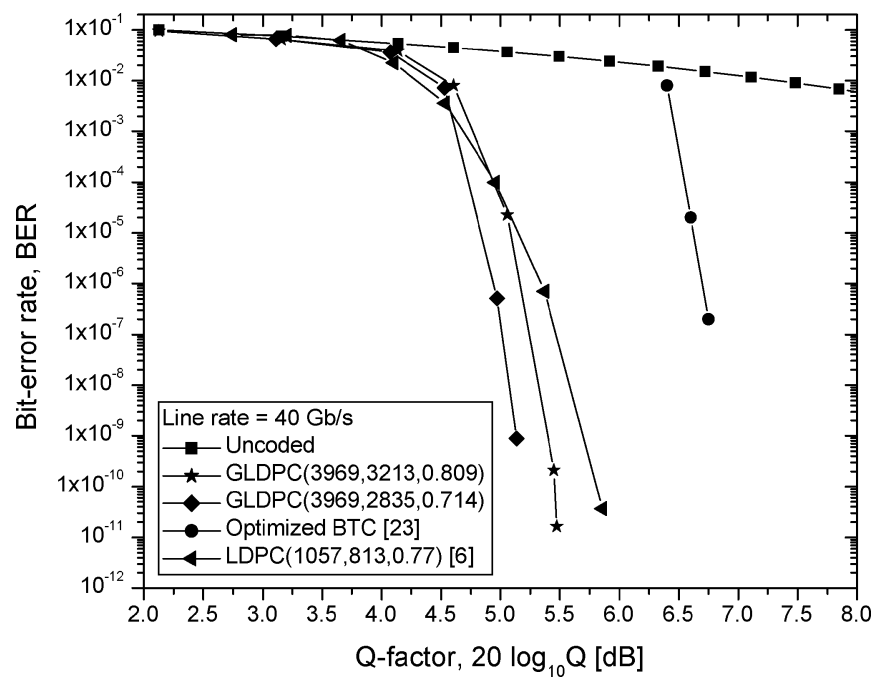

Fig. 3. BER performance of GLDPC codes with Hamming $(63,57)$ constituent code at $40 \mathrm{~Gb} / \mathrm{s}$ (line rate) (ten iterations).

therefore in a similar environment as simulated in this work. It also outperforms the best LDPC code proposed so far for optical communications, our proposal [6] (in terms of coding gain). As expected, GLPDC decoder with three supercodes$\operatorname{GLDPC}(3969,2835,0.714)$ performs better than that with two supercodes-GLDPC $(3969,3213,0.809)$ for the same constituent Hamming $[63,57,3]$ code. By reducing the code rate the performance can be improved further (Fig. 2), at the price of reducing the information rate.

Results of simulations for GLDPC code for which a LDPC code of column-weight 3 is used as a global code are shown in Fig. 4 for two different constituent codes: Hamming [63,57,3] with BCJR decoder (diamond curve) or modified MPA decoder [24] (square curve) and LDPC $(90,100)$ (designed using the codes we proposed in [7]) with MPA in decoding (circle curve). Although the LDPC code of minimum distance of at least 11 is used as a local code and MPA in decoding, the GDLPC code based on Hamming codes with a BCJR decoding algorithm performs better. On the other hand, the MPA based solution is a much

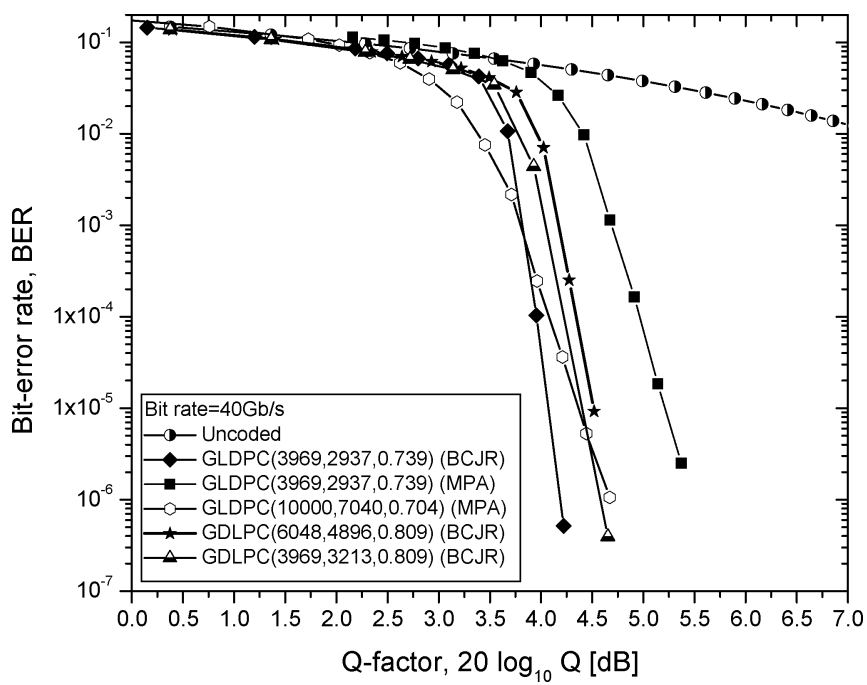

Fig. 4. BER performance of GLDPC codes for global code being LDPC and local code being either Hamming (diamond and square curves) or LDPC (circle curve) code, at bit rate of $40 \mathrm{~Gb} / \mathrm{s}$.

more cost-effective solution than the BCJR based solution. By employing the modified MPA [24] instead of BCJR we are losing about $1.25 \mathrm{~dB}$, but the implementation of Hamming [63,57,3] soft decoder using modified MPA is significantly simpler.

The $[6048,4896,0.809]$ GLDPC code ("star curve" in Fig. 4), designed using Theorem 2, performs the best for a given overhead of $23.53 \%$ and provides a coding gain of almost $12 \mathrm{~dB}$.

This result is likely a consequence of the large girth of the global LDPC code, and the large overall minimum distance of the GLDPC code. Notice that the codes shown in Figs. 2 and 3 are not directly comparable with codes in Fig. 4, since the bit rate in the latter case is lower than $40 \mathrm{~Gb} / \mathrm{s}$. Choosing a bit rate of $40 \mathrm{~Gb} / \mathrm{s}$ for a code of rate 0.677 results into an unacceptably high line rate of $59.08 \mathrm{~Gb} / \mathrm{s}$.

Another interesting observation is that the increase in the line rate introduces more nonlinearities, and consequently, the signal is more degraded. In order to keep the same value for the Q-factor, the optical signal-to-noise ratio has to be increased, resulting in improved BER performance. 


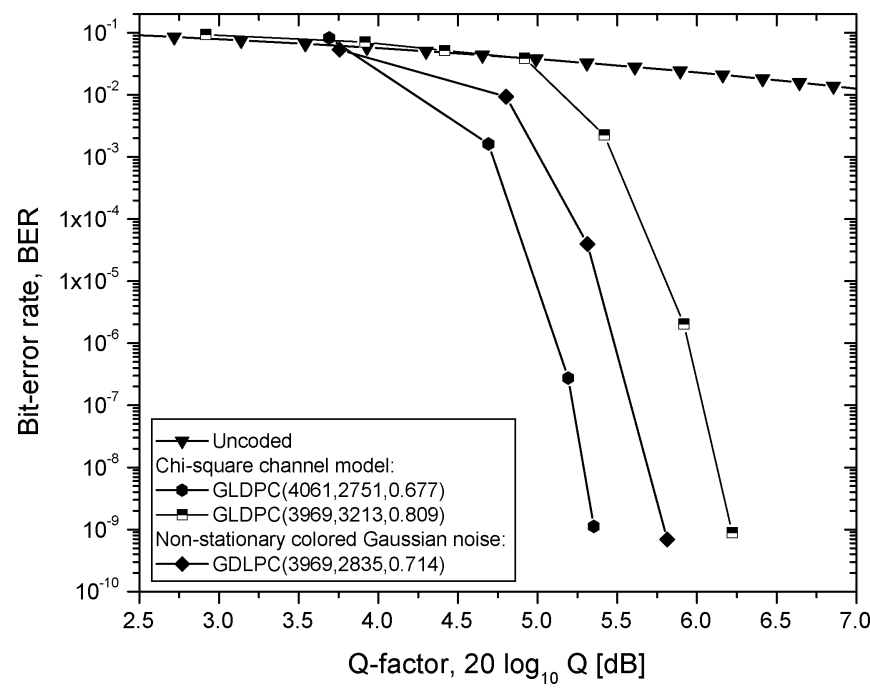

Fig. 5. BER performance of GLDPC codes for chi-square and nonstationary colored Gaussian channel models.

The results of simulations for chi-square [32] (in tenth iteration) and nonstationary colored Gaussian channel model [19] at line rate of $40 \mathrm{~Gb} / \mathrm{s}$ are shown in Fig. 5. The prediction of BER performance of GLDPC code from nonstationary Gaussian channel model is better than chi-square, and can be used as a starting point for initial prediction of the FEC performance.

Based on our previous results [6], [7], as well as the recent results by Koetter [34] and Richardson [35], we believe that the proposed GLDPC codes will not experience the error floor phenomena. However, the existence of a BER floor of LDPC/GLDPC codes is still an open issue, although there has been some progress recently [34], [35].

\section{CONCLUSION}

We proposed a novel class of FEC for optical transmission systems, the class of GLDPC codes. GLDPC codes, the generalization of both LDPC and turbo codes, demonstrate largest so far reported coding gain of almost $12 \mathrm{~dB}$ for $23.6 \%$ of overhead (at BER below $10^{-12}$ ).

The excellent BER performance of GLPDC codes in the presence of ASE noise, fiber nonlinearities, chromatic dispersion, and inter-symbol interference selects them as a very promising option for next generation of optical transmission systems and for network applications.

\section{REFERENCES}

[1] K. Azadet et al., "Equalization and FEC techniques for optical transceivers," IEEE J. Solid-State Circuits, vol. 37, no. 3, pp. 317-327, Mar. 2002.

[2] C. Howland and A. Blanksby, "A $220 \mathrm{~mW} 1 \mathrm{~Gb} / \mathrm{s}$ 1024-bit rate-1/2 low density parity check code decoder," in IEEE Conf. Custom Integrated Circuits, 2001, pp. 293-296.

[3] — "A 690-mW 1-Gb/s 1024-b, rate-1/2 low-density parity-check code encoder," IEEE J. Solid-State Circuits, vol. 37, no. 3, pp. 404-412, Mar. 2001.

[4] T. Richardson and R. Urbanke, "The renaissance of Gallager's lowdensity parity-check codes," IEEE Commun. Mag., vol. 41, no. 8, pp. 126-131, Aug. 2003.

[5] T. Mizouchi et al., "Forward error correction based on block turbo code with 3-bit soft decision for $10 \mathrm{~Gb} / \mathrm{s}$ optical communication systems," IEEE J. Sel. Topics Quantum Electron., vol. 10, no. 2, pp. 376-386, Mar./Apr. 2004.
[6] I. B. Djordjevic, S. Sankaranarayanan, and B. Vasic, "Projective-plane iteratively decodable block codes for WDM high-speed long-haul transmission systems," J. Lightw. Technol., vol. 22, no. 3, pp. 695-702, Mar. 2004.

[7] I. B. Djordjevic and B. Vasic, "MacNeish-Mann theorem based iteratively decodable codes for optical communication systems," IEEE Commun. Lett., vol. 8, no. 8, pp. 538-540, Aug. 2004.

[8] W. E. Ryan, "An introduction to LDPC codes," in CRC Handbook for Coding and Signal Processing for Recording Systems, B. Vasic and E. M. Kurtas, Eds. Boca Raton, FL: CRC, 2005.

[9] R. M. Tanner, "A recursive approach to low complexity codes," IEEE Trans. Inf. Theory, vol. IT-27, no. 5, pp. 533-547, Sep. 1981.

[10] J. Boutros, O. Pothier, and G. Zemor, "Generalized low density (Tanner) codes," in Proc. 1999 IEEE Int. Conf. Communication (ICC 1999), vol 1, 1999, pp. 441-445.

[11] M. Lentmaier and K. S. Zigangirov, "On generalized low-density paritycheck codes based on Hamming component codes," IEEE Commun. Lett., vol. 3, no. 8, pp. 248-250, Aug. 1999.

[12] O. Pothier, L. Brunel, and J. Boutros, "A low complexity FEC scheme based on the intersection of interleaved block codes," in Proc. 49th IEEE Vehicular Technol. Conf., vol. 1, 1999, pp. 274-278.

[13] T. Zhang and K. K. Parhi, "High-performance, low-complexity decoding of generalized low-density parity-check codes," in Proc. IEEE Global Telecommunications Conf. 2001 (IEEE GLOBECOM), vol. 1, 2001, pp. $181-185$.

[14] — "A class of efficient-encoding generalized low-density paritycheck codes," in Proc. IEEE Int. Conf. Acoustics, Speech, Signal Processing (ICASSP 2001), vol. 4, 2001, pp. 2477-2480.

[15] T. M. N. Ngatched and F. Takawira, "Efficient decoding of generalized low-density parity-check codes based on long-component codes," in Proc. Wireless Communication Networking Conf. (WCNC 2003), vol. 1, 2003, pp. 705-710.

[16] S. Hirst and B. Honary, "Application of efficient chase algorithm in decoding of generalized low-density parity-check codes," IEEE Commun Lett., vol. 6, no. 9, pp. 385-387, Sep. 2002.

[17] L. R. Bahl, J. Cocke, F. Jelinek, and J. Raviv, "Optimal decoding of linear codes for minimizing symbol error rate," IEEE Trans. Inf. Theory, vol. 20, no. 2, pp. 284-287, Mar. 1974.

[18] A. Ashikhmin and S. Litsyn, "Simple MAP decoding of first order Reed-Muller and Hamming codes," IEEE Trans. Inf. Theory, vol. 50, no. 8, pp. 1812-1818, Aug. 2003.

[19] I. B. Djordjevic and B. Vasic, "An advanced direct detection receiver model," J. Opt. Commun., vol. 20, pp. 6-9, Feb. 2004.

[20] F. McWilliams and N. Sloane, The Theory of Error Correcting Codes. New York: North Holland, 1977.

[21] V. Nagarajan, N. Jayakumar, S. Khatri, and O. Milenkovic, "Highthroughput VLSI implementations of iterative decoders and related code construction problems," in Proc. IEEE GLOBECOM 2004, vol. 1, Dec. 2004, pp. 361-365.

[22] R. G. Gallager, Low-Density Parity-Check Codes. Cambridge, MA: MIT, 1963.

[23] O. A. Sab and V. Lemarie, "Block turbo code performances for long-haul DWDM optical transmission systems," in Proc. Optical Fiber Communication Conf. (OFC), vol. 3, 2001, pp. 280-282.

[24] S. Sankaranarayanan and B. Vasic, "Iterative decoding of linear block codes," IEEE Trans. Inf. Theory, submitted for publication.

[25] S. Dolinar and D. Dilvasar, "Weight Distributions for Turbo Codes using Random and Nonrandom Permutations," Progr. Rep., JPL TDA, 42-122, 1995.

[26] N. Ehtiati et al., "Improved interleaver design for turbo coded intersymbol interference channels," in Proc. IEEE 58th Conf. Vehicular Technol. (VTC 2003), Oct. 2003, pp. 327-331.

[27] H. Zhang and J. Moura, "The design of structured regular LDPC codes with large girth," in Proc. IEEE GLOBECOM 2003, vol. 7, 2003, pp. 4022-4027.

[28] O. Milenkovic and S. Laendner, "Analysis of the cycle-structure of LDPC codes based on Latin squares," in Proc. 2004 IEEE Int. Conf. Communications, vol. 2, Jun. 2004, pp. 777-781.

[29] R. Lidle and H. Niederreiter, Finite Fields, CA: Academic Press, 1997.

[30] J. Fan, "Array codes as LDPC codes," in Proc. 2nd Int. Symp. Turbo Codes, 2000, pp. 543-546.

[31] A. Ashikhmin, G. Kramer, and S. ten Brink, "Extrinsic information transfer functions: Model and erasure channel properties," IEEE Trans. Inf. Theory, vol. 50, no. 11, pp. 2657-2673, Nov. 2004.

[32] P. A. Humblet et al., "On the bit error rate of lightwave systems with optical amplifiers," J. Lightw. Technol., vol. 9, no. 11, pp. 1576-1582, Nov. 1991. 
[33] I. B. Djordjevic and B. Vasic, "High code rate low-density parity-check codes for optical communication systems," IEEE Photon. Technol. Lett., vol. 16, no. 6, pp. 1600-1602, Jun. 2004.

[34] R. Koetter, "Iterative coding techniques, pseudocodewords, and their relationship," presented at the LANL Workshop on Applications of Statistical Physics to Coding Theory, Santa Fe, NM, Jan. 10-12, 2005.

[35] T. Richardson, "Error floors of LDPC codes," presented at the 41st Allerton Conf. Communications, Control and Computing, Allerton, IL, Oct. 2003.

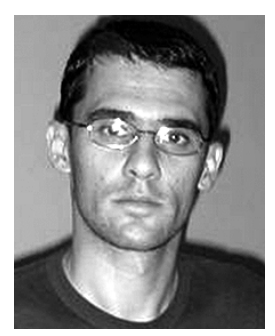

Ivan B. Djordjevic (M'03) received the Dipl.Ing., M.S., and Ph.D. degrees, all in electrical engineering, from the University of Nish, Nish, Serbia, in 1994, 1997, and 1999, respectively.

From 1994 to 1996, he was with the Faculty of Electronic Engineering, University of Nish, working on modeling and simulation of optical/digital communication systems. From 1996 to 2000, he was with the State Telecommunications Company (Serbia Telecom), District Office for Networks, Nish, Serbia, where he was involved in digital transmission systems commissioning and acceptance, design, maintenance, installation, and connection. From 2000 to 2001, he was with the National Technical University of Athens, Greece, and with TyCom US, Inc. (now TyCo Telecommunications), USA, where he was involved in modeling and simulation of dense-wavelength-division-multiplexing (DWMD) systems. During 2002 and 2003, he was with the University of Arizona, Tucson; the University of Bristol, U.K.; and the University of West of England, Bristol, U.K., working on forward-error correction and iterative decoding for optical transmission, optical CDMA, high-speed transmission, and optical switches. $\mathrm{He}$ is now with the University of Arizona, on leave from the University of West of England. He is an author of more than 35 international journal papers and more than 45 international conference papers. His research interests include DWDM fiber-optic communication systems and networks, FEC for optical communications, optical CDMA, optical packet switching, coding theory, coherent optical communications, and statistical communication theory.
Olgica Milenkovic (M'03) received the M.S. degree in mathematics and the Ph.D. degree in electrical engineering from the University of Michigan, Ann Arbor, in 2001 and 2002, respectively.

She is currently an Assistant Professor in the Department of Electrical and Computer Engineering at the University of Colorado, Boulder. Her research interests are in the area of error-control coding, constrained coding, probability theory, analysis of algorithms, and genetics.

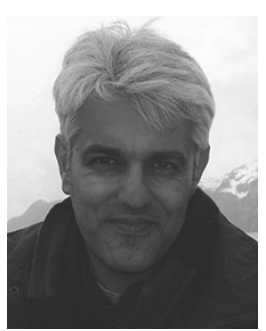

Bane Vasic (S'92-M'93-SM'02) received the B.S., M.S., and Ph.D. degrees in electrical engineering from the University of Nish, Serbia, Yugoslavia, in 1989, 1991, and 1994, respectively.

From 1996 to 1997, he was a Visiting Scientist at the Rochester Institute of Technology and Kodak Research, both in Rochester, NY, where he was involved in research on optical storage channels. From 1998 to 2000, he was with Lucent Technologies, where he was involved in research coding schemes and architectures for high-speed applications. He was also involved in iterative decoding and low-density parity check codes, as well as development of codes and detectors for five generations of Lucent (now Agere) chips. Currently, he is a Faculty Member of the Electrical and Computer Engineering Department, University of Arizona, Tucson. He has authored more than 25 journal articles, more than 50 conference papers, and more than six book chapters and one book. His research interests include coding theory, information theory, communication theory, digital communications, and recording.

Dr. Vasic is a Member of the Editorial Board of the IEEE TRANSACTIONS ON MAGNETICs. He served as Technical Program Chair of the IEEE Communication Theory Workshop 2003 and as Co-Organizer of the Center for Discrete Mathematics and Theoretical Computer Science (DIMACS) Workshops on Optical/Magnetic Recording and Optical Transmission and Theoretical Advances in Information Recording, 2004. 